\title{
Response to 'Comment on: Optimisation of intravitreal injection technique using a Barraquer speculum with solid flat blade and finger stabilisation'
}

\author{
Craig Wilde ${ }^{1} \cdot$ Mary Awad ${ }^{2} \cdot$ Ali Poostchi $\mathbb{i}^{1} \cdot{\text { Andrew } \text { Ross }^{1} \cdot \text { Winfried M Amoaku (i) }}^{1}$
}

Received: 9 October 2018 / Accepted: 10 October 2018 / Published online: 24 October 2018

(c) The Royal College of Ophthalmologists 2018

We thank the authors for their interest in our technique and congratulate them on the development of their device, which we expect will make a useful contribution to the safe delivery of intravitreal injections (IVT) [1]. Such a device offers distinct safety advantages, particularly with reference to stabilising the globe, determining the point of injection from the limbus, as well as the angle and depth of injection, making the technique user friendly.

We have seen several cases of lens and retinal trauma from IVT over the years, and such a device should hopefully reduce the incidence of these uncommon, but significant problems.

For the majority of patients and trained surgeons the traditional IVT procedure is not difficult or dangerous, and special devices are not required. Reluctance to trial these new products may limit their adoption and widespread use. A comparison against the traditional IVT technique may be required to assess both patient and surgeon preference.

Establishing definite advantages of such IVT devices, including more comfort for patients, increased speed of procedure, or indeed reduced real- world cost, will more likely lead to wider adoption, especially when established instrumentation and techniques like ours are readily available in most eye units.

For now, while devices like the one described here (BVI Malosa), or the InVitrea Injection Assistant (FCI Ophthalmics), have limited uptake across the UK, we hope that people including the non-medical injectors will find our technique for globe stabilisation useful.

\section{Compliance with ethical standards}

Conflict of interest The authors declare that they have no conflict of interest.

\section{References}

1. Waqar S. Comment on: Optimisation of intravitreal injection technique using a Barraquer speculum with solid flat blade and finger stabilization. Eye. 2018. Epub ahead of print. https://doi.org/ 10.1038/s41433-018-0248-3.
Neelakshi Bhagat

bhagatne@njms.rutgers.edu

1 Ophthalmology and Vision Sciences, Division of Clinical Neurosciences, B Floor, EENT Centre, Queen's Medical Centre, University of Nottingham, Nottingham, UK

2 University Hospital, Queen's Medical Centre, Nottingham, UK 